\title{
Electrical Detection and Control System Based on Artificial Intelligence
}

\author{
Ren $\mathrm{Li}^{*}$, Hang Zhou \\ Henan Polytechnic Institute, Nanyang, China \\ *Corresponding Author.
}

\begin{abstract}
With the rapid development of society, many industries have integrated intelligent advanced technology, and electrical automation control is one of them. Electrical automation control system is a control system for the efficiency, status, quality and other aspects of electrical operation. Through the system, electrical operation can be well controlled. After the use of intelligence, the system has the ability of sub identification and decisionmaking. This paper first briefly analyzes the application theory of artificial intelligence, and then expounds the advantages of artificial intelligence controller. This paper studies the application of artificial intelligence technology in electrical automation control system from two aspects of the application of artificial intelligence in $D C$ drive and AC drive, and analyzes the advantages of artificial intelligence control. Taking the application of AI Artificial Intelligence regulator in the control of an electrical appliance as an example, this paper expounds the circuit principle, implementation scheme and application effect of the system.
\end{abstract}

Keywords: Electrical automation, control system, operation efficiency, artificial intelligence regulator.

\section{Introduction}

The ESANN method, which combines artificial neural network with expert system, is put forward based on DGA. ESANN takes advantage of the advantages of neural network and expert system, and makes deep improvement on this basis [1-2]. Power transformer is an important equipment in power system, and the diagnosis of its abnormal situation is very important for the safety and reliability of the whole power system. Improving the level of transformer operation and maintenance and technical management has become an important means to ensure the reliability of power supply.

As we all know, transformer fault diagnosis is a very complex problem. Many factors, such as transformer capacity, voltage level, insulation performance, working environment, operation history, and even products from different manufacturers, will affect the diagnosis results [3]. All kinds of test methods and test instruments only play the role of obtaining transformer operation status information, and making correct judgment based on these information must rely on rich operation and maintenance experience. Due to the important role of human experience in this problem, it is of great practical significance to develop intelligent fault diagnosis system by using artificial intelligence technology [4-5].

Expert system (ES) and artificial neural network (ANN) are two research fields at present, but there is a "bottleneck" of knowledge acquisition in expert system technology [6-7]:

(1) Most of ES knowledge base acquisition comes from manual transplantation. Experts transplant knowledge to computer, which is time-consuming and laborious;

(2) The knowledge base of ES can only solve the problems in this field, but the ability to solve the problems in similar fields is very poor;

(3) They have the ability to write es, but they have no self-organizing memory and self-learning ability, so they can not be upgraded in practical application;

(4) The larger the knowledge base, the slower the processing speed and the lower the efficiency, but the accuracy

ISSN: 0010-8189

(C) CONVERTER 2020 
and efficiency can not be both.

Because of its self-organization and self-learning ability, ANN can overcome the shortcomings of traditional ES in this aspect. By using DGA technology, ESANN has been applied in transformer fault diagnosis. In many ANN models, BP network is suitable for pattern recognition and fault diagnosis because of its good ability of pattern classification.

\section{ESANN system flow}

2.1 ESANN system structure block diagram

The ESANN system shown in fig. 1 includes the following parts [8-12]:

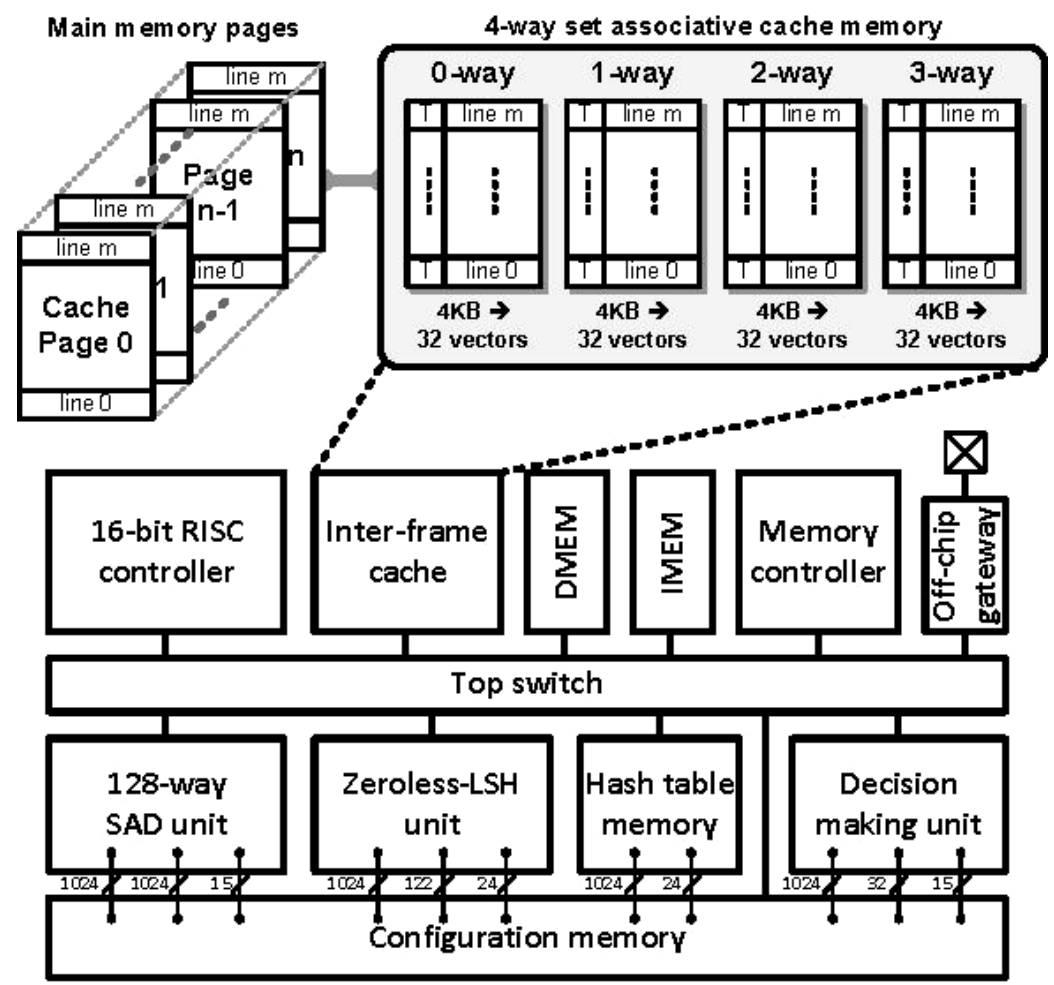

Fig 1: ESANN process

(1)ANN detection, the detection result is normal or abnormal, which provides a basis for further detection;

(2)ES detection, the detection result is normal or abnormal, which provides a basis for further detection;

(3) Fault analysis using ANN;

(4) Fault analysis using ES;

(5) Comprehensive logic synthesis of fault diagnosis results;

(6) Give maintenance suggestions to the system, estimate the interval of the next test, and realize correct maintenance actions.

\subsection{Input data}

Input data includes the following aspects :

ISSN: 0010-8189 
(1) The dissolved gas content ( $\mu \mathrm{l} / \mathrm{l}$ ) and gas production rate ( $\mathrm{ml} / \mathrm{h}$ ), including $\mathrm{CH}_{4}, \mathrm{C}_{2} \mathrm{H}_{4}, \mathrm{CO}, \mathrm{CO}_{2}, \mathrm{H}_{2}, \mathrm{C}_{2} \mathrm{H}_{6}$, $\mathrm{C}_{2} \mathrm{H}_{2}, \mathrm{O}_{2}, \mathrm{C}_{2} \mathrm{H}_{2}, \mathrm{~N}_{2}$, are the main input parameters of the diagnosis process.

(2) Oil sample information, including date sample (used to compare with historical gas production trend) and location of gas sample (used to determine the weight of relevant gas).

(3) Relevant information of transformer, including transformer capacity, voltage grade, core type, number of grades, insulation performance, working environment and operation history.

(4) Background information, including transformer serial number, substation information, number of identified oil samples, historical data of fault report, etc.

Although the dissolved gas in transformer oil is affected by many factors, only a small part can be used for fault diagnosis. At the same time, in order to update the system database in time to obtain new diagnostic rules, it is very important to save as much information as possible in the database.

\subsection{Logic output of diagnosis}

The output of diagnosis results includes fault types, reliability of diagnosis results, re inspection time and maintenance suggestions:

(1) Superheat $(\mathrm{OH})$;

(2) Oil overheating $(\mathrm{OHO})$;

(3) Low energy discharge (LED);

(4) High energy discharge or arc (HEDA);

(5) Cellulose insulation damage (CD)

\subsection{Establishment of database}

In the process of ESANN diagnosis, every sample is recorded in a dynamic database, which is convenient to use as a training database next time. Diagnosis is also based on this record, and the diagnosis results are stored in another dynamic database, which is convenient to compare with historical records and update diagnosis rules in time.

\section{Working principle of ESANN}

In ESANN system, there will be four independent ANN units used for fault detection, and the ann. BP network with three hidden layers trained by BP algorithm is adopted, that is, the Back. Propagation learning theory of forward multilayer neural network (abbreviated as BP, which was first proposed by Weber in 1974).

Among many ANN types, the BP network which uses the Error Back. Propogation algorithm as the training algorithm has a wide application prospect in the fault diagnosis system because of its good pattern classification ability and is especially suitable for fault diagnosis.

ESANN is written in Microsoft Visual C++, and each module of ESANN is introduced in detail below.

\subsection{Normal or abnormal detection based on ANN}

The input data are the fault gas content $(\mu \mathrm{l} / \mathrm{l})$ and gas production rate $(\mathrm{ml} / \mathrm{h})$. Two ANN networks are used to judge whether it is normal, as shown in Figure 2.

ISSN: 0010-8189 


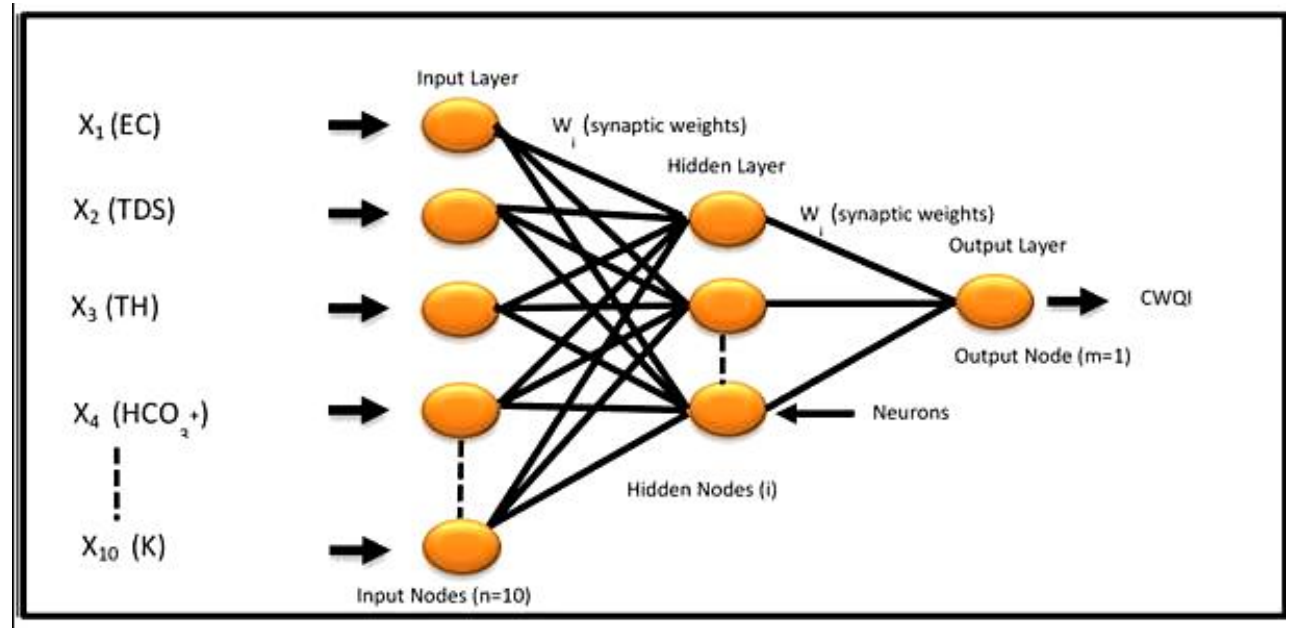

Fig 2: Normal or abnormal detection based on ANN

The input data goes through two independent detection links. Only when the outputs of the two links are normal, the output is "normal", otherwise the output is "abnormal".

3.2 Normal or abnormal detection based on ES

The normal or abnormal detection based on ES is shown in Figure 3.

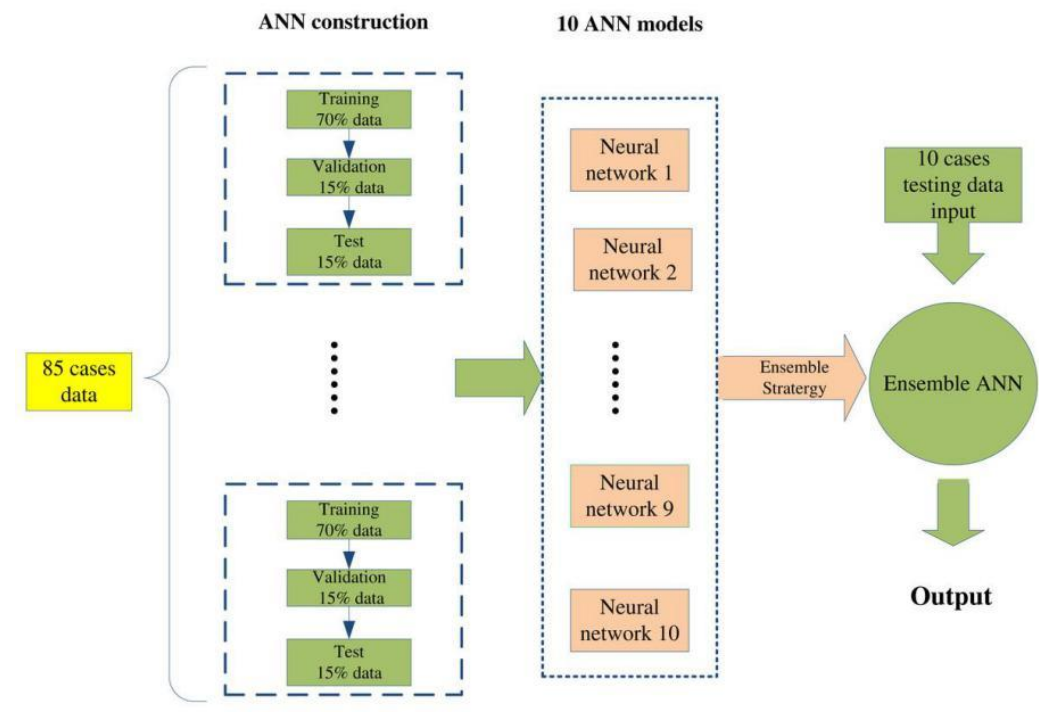

Fig 3: Normal or abnormal detection based on ES

The input data goes through four independent detection links respectively. Only when the output of the four links is normal, the output is "normal", otherwise the output is "abnormal". The criteria of the four detection links are: whether the fault gas is within the limit value (using IEEE C57.104 standard); three ratio method: absolute gas production rate; relative gas production rate.

\subsection{Fault detection based on ANN}

When the result of the above judgment is "abnormal", the data is sent to ANN and ES respectively for fault detection and judgment, as shown in Figure 4.

ISSN: 0010-8189 


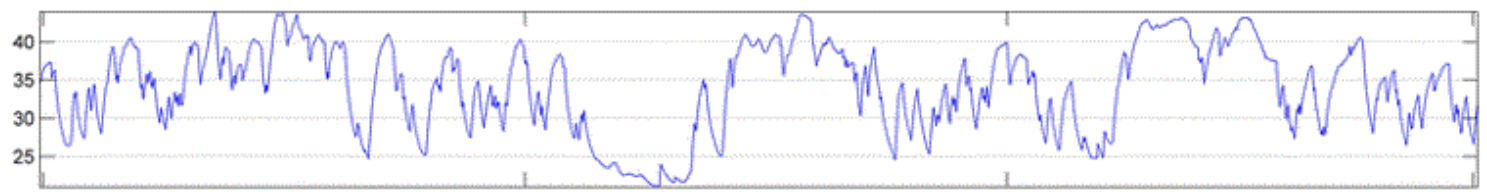

(a) Turbine bearing temperature $(t-1)$

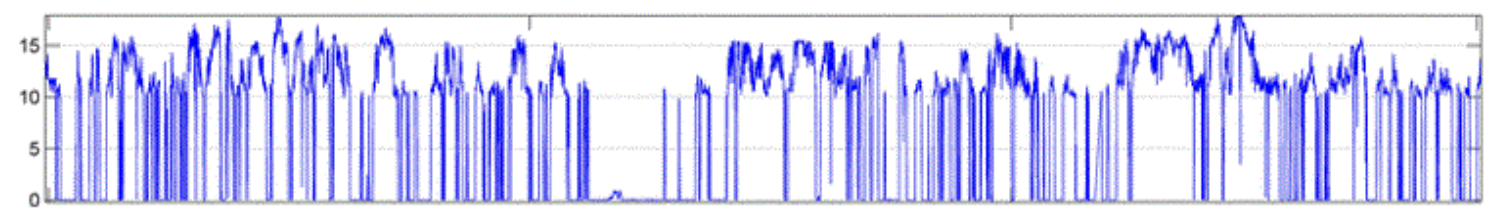

(b) Turbine speed

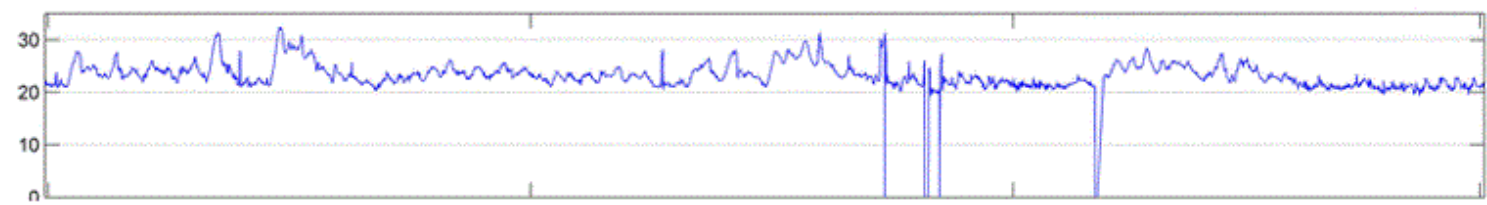

(c) Nacelle temperature ( $t-1)$

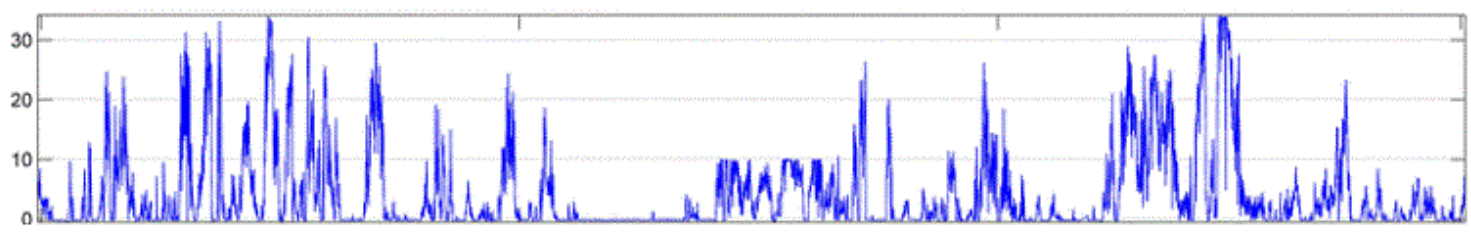

(d) Active power

Fig 4: Fault detection results based on ANN

In the ANN detection link, two independent Ann links are used to judge the main faults respectively. In a large number of previous studies done by the author, this structure has higher diagnosis accuracy than the single ANN network output in Figure 2, and the sample training time will be greatly shortened.

\section{Examples and discussions}

The system discussed in this paper is a sample trained by 220 sets of data, including 22 normal cases, of which 150 sets of data are used to train ANN network and 70 sets of data are used as experimental tests. Table 1 shows the accuracy comparison between ESANN, ANN and ES when running alone.

Table 1 Test results of training samples \%

\begin{tabular}{|c|c|c|c|c|c|c|}
\hline \multirow{2}{*}{ Fault type } & \multicolumn{2}{|c|}{ ESANN } & \multicolumn{2}{c|}{ ANN } & \multicolumn{2}{c|}{ ES } \\
\cline { 2 - 7 } & Train & Test & Train & Test & Train & Test \\
\hline Overheated & 100 & 97 & 100 & 95 & 97.3 & 90 \\
\hline Oil overheating & 100 & 95 & 100 & 95 & 99.3 & 90 \\
\hline Low energy discharge & 100 & 95 & 100 & 95 & 95.2 & 91 \\
\hline High energy discharge or arc & 99.7 & 97 & 100 & 95 & 96.7 & 85 \\
\hline Cellulose insulation damaged & 99.7 & 93.3 & 100 & 95 & 90.7 & 91.7 \\
\hline
\end{tabular}

ISSN: 0010-8189 
Es judges that there is no CD fault, but there may be HEDA fault, because the characteristic gas CO of CD fault is lower than its limit and there is a small amount of $\mathrm{C}_{2} \mathrm{H}_{2}$ (characteristic gas of HEDA fault). But in this case, because ANN is not simply based on the limit value to judge, but through the adaptive reasoning process to get the results, so it has good diagnostic accuracy.

The diagnostic results of ES showed that there were $\mathrm{OH}$, oho and $\mathrm{CD}$ faults, because the characteristic gases $\mathrm{CH}_{4}$, $\mathrm{C}_{2} \mathrm{H}_{4}, \mathrm{CO}$ and cah6 were obviously excessive. When Ann judged the CD fault, these characteristic gases were not obviously excessive, which may be due to the lack of similar samples.

ES did not judge the existence of LED fault, but thought that HEDA fault may exist, which may be due to the gas production rate not reaching the lower limit of LED fault judgment value and a small amount of $\mathrm{C}_{2} \mathrm{H}_{2}$. At the same time, because there is excessive $\mathrm{H}_{2}$ and the content of other combustible gases is very small, ANN judges the LED fault and eliminates the possibility of HEDA fault. The output result of ESANN is correct.

In our testing process, it happened that both ANN and ES judged correctly, but the comprehensive output result was incorrect. This kind of situation is very rare, so we will make further follow-up study.

To sum up, the diagnostic accuracy of ESANN system is obviously better than that of ES and ANN alone.

\section{Advantages of artificial intelligence in electrical engineering automation}

The work of traditional controller to adjust parameters is complex, it is difficult to master the adjustment operation, and the adaptability is weak. The emergence of artificial intelligence not only makes up for the defects of the traditional controller in adjusting parameters, but also has a more prominent advantage that it does not rely on the participation of professional personnel in the field to complete the whole process of parameter setting independently, that is, according to the reasonable parameter data, combined with the use of language and response information. In addition, it can also make simple modification after parameter setting to make it better adapt to the actual situation

The application of artificial intelligence in electrical engineering automation controller eliminates the influence of uncertain factors such as the change of model setting parameters and different numerical types in the process of calculation when the traditional controller is building the model.

Some uncertain factors are difficult to affect artificial intelligence technology, its ability to resist interference is very strong. The operating system can hardly change the parameters set before operation, and they always keep a fixed value. Therefore, the error between the actual value and the theoretical value is very small.

Compared with the traditional electrical engineering automation control technology which has good control effect on specific objects and unstable control effect on other objects, the electrical controller integrated with artificial intelligence technology has a strong consistency. It can better evaluate any unknown data input into the system, and the performance consistency between products is also guaranteed.

A variety of electrical equipment such as lines, machines, transformers and various wires and cables have been applied to the operation of electrical engineering. The placement of these equipment is chaotic. In addition, the cleaning of various machines also requires a lot of manpower. However, the application of artificial intelligence to electrical engineering automation can effectively solve this thorny problem. There is no machine to clean, which reduces the demand for machine cleaning personnel. In this way, the input of human and material resources are greatly reduced.

\section{Conclusion}

ANN adopts a three-layer hidden network structure with the best performance for single fault diagnosis. By selecting appropriate methods for sample training, it has higher diagnostic reliability and accuracy, and can be retrained by new sample experience values. The comprehensive fault diagnosis output makes use of the advantages of ANN and Es, and the test results show that ESANN can get more accurate diagnosis results than

ISSN: 0010-8189 
ES and ANN when used alone.

The combination of expert system and artificial neural network is the only way to simulate human intelligent thinking, but the development of them is a long and gradual process, which needs continuous innovation and improvement.

\section{References}

[1] Wang Jinliang. Research on the Application of Artificial Intelligence Technology in Electrical Automation Control System. Guide to Becoming Rich in Science and Technology, 2012, 30: 315-316

[2] Song Lai. Design and Implementation of Mine Electrical Automation Control System Based on Computer and Artificial Intelligence Technology. China Metal Bulletin, 2016 (11): 80-81

[3] Liu Xiangyang, Wang Dan. Design and Research of Electrical Automation Control System Based on Intelligent Technology. Electronic Design Engineering, 2019, V.27; No.414 (16): $72-75$

[4] Wen Wenhao. Research on Electrical Automation Control System Based on Artificial Intelligence. Information and Computer (theoretical Edition), 2020, V.32; No.446 (04): 152-153

[5] Liu Xiaoyan. Application of Artificial Intelligence Technology in Electrical Automation Control System. Rural Economy and Technology, 2019, 030 (022): 254-255

[6] Guo Yanan, Wang Yanfei. Application of Artificial Intelligence Technology in Electrical Automation Control. Architectural Engineering Technology and Design, 2018, 16: 4184

[7] Huang Qiang. Application of Artificial Intelligence Technology in Electrical Automation Control. Consumer Guide, 2019, 10: 120

[8] Yin Zhangsheng. Research on Electrical Automatic Control Technology Under Artificial Intelligence Technology. Development Orientation of Building Materials, 2019, 017 (009): 363

[9] Wang Li. Application of Artificial Intelligence in Electrical Control System. Full Text Edition: Engineering Technology, 2016 (6): 175-175

[10] Jiang Fkuan, Zhou Rui. Discussion on the Application of Artificial Intelligence Technology in Electrical Automation Control. Dual Use Technologies and Products, 2018, 6: 96

[11] Yao Linhu. Application of Artificial Intelligence in Electrical Automation Control. Intelligent City Application, 2020, 3 (8): 91

[12] Wang Huiqian. Application and Development of Artificial Intelligence Technology in Electrical Control System. Journal of College of Electronic Engineering, 2019, 008 (010): P.137-137 